\title{
PERCEPCIÓN DE ENFERMERAS Y FAMILIARES DE PACIENTES SOBRE CONDUCTAS DE CUIDADO IMPORTANTES
}

\author{
PERCEPTION OF NURSES AND FAMILIES OF PATIENTS \\ REGARDING IMPORTANT CARE BEHAVIORS
}

\author{
Bárbara Andrea Salas SaAvedra * \\ María Alejandra Galiano Gálvez ${ }^{* *}$
}

\begin{abstract}
RESUMEN
Objetivo: Determinar si existen diferencias entre las enfermeras de pacientes hospitalizados y sus familiares, respecto de la percepción de cuáles conductas de cuidado son importantes. Material y método: Estudio descriptivo, transversal, cuya muestra, estratificada por servicio, estuvo constituida por 54 enfermeras y 96 familiares de un hospital general. Para medir la variable respuesta se empleó el Caring Assessment Instrument (Care- Q) de Patricia Larson, que consta de 50 preguntas dividas en 6 subescalas que miden el comportamiento del profesional de enfermería en la entrega de cuidado: a) es accesible, b) explica y facilita, c) conforta, d) se anticipa, e) mantiene relación de confianza y f) monitorea y hace seguimiento. Resultados: La priorización de la primera conducta, representada por la subescala "monitorea y hace seguimiento", coincide en ambos grupos. En relación a la importancia asignada al resto de las conductas de cuidado y sus subescalas, se observan diferencias en ambos grupos. Conclusión: La priorización de las categorías y conductas de cuidado entre las enfermeras y los familiares de los pacientes son diferentes y como primera selección se enfocan hacia conductas del tipo técnicas, más que al desarrollo de habilidades sociales.
\end{abstract}

Palabras clave: Conductas del cuidado, percepción, enfermeras, pacientes, familia.

\begin{abstract}
Objective: To determine whether there are differences between nurses of hospitalized patients and their relatives, regarding the perception they have of what care behaviors are important. Material and method: A cross-sectional descriptive study whose sample, stratified by service, consisted of 54 nurses and 96 relatives of a general hospital. To measure the response variable, the Caring Assessment Instrument (Care- Q) by Patricia Larson was used, which consists of 50 questions divided into 6 sub-scales that measure the behavior of the nursing professional in the delivery of care: a) it is accessible, b) explains and facilitates, c) comforts, d) anticipates, e) maintains a relationship of trust and f) monitors and follows up. Results: The prioritization of the first behavior, represented by the subscale "monitors and follows-up", coincides in both groups. Regarding the importance assigned to the rest of the care behaviors and their subscales, differences are observed in both groups. Conclusion: The prioritization of care categories and behaviors between nurses and patients' relatives are different and, as a first choice, it focuses on technical type behavior rather than the development of social skills.
\end{abstract}

Key words: Caring behavior, perception, nurses, patients, family.

Fecha recepción: 27/04/15 Fecha aceptación: 24/10/16

Enfermera. Supervisora UCI, Hospital de Carabineros del General Humberto Arriagada. Santiago, Chile. Email: bsalassaavedra@gmail.com

${ }^{* *}$ Enfermera. Docente Escuela de Enfermería, Universidad de los Andes. Santiago, Chile. Email: magalianog@uandes.cl 


\section{INTRODUCCIÓN}

El ingreso de un paciente a un hospital implica un gran desafío, tanto a nivel personal como familiar. Si bien la enfermera trabaja directamente con el paciente, la familia es un componente muy importante en la hospitalización de este, ya que es una situación estresante que genera desequilibrio en la vida habitual de toda persona. El impacto que sufre el paciente y su familia debe ser aminorado por el cuidado que entrega la enfermera, quien debe brindar herramientas que permitan recuperar el bienestar físico y emocional alterado por la hospitalización. La enfermera deberá ser un vehículo facilitador en la expresión de sentimientos y emociones de todos aquellos que se involucran en momentos de enfermedad, permitiendo y facilitando a posterior, la re-inserción en la vida cotidiana de cada uno de ellos. Así, las enfermeras, a través de las conductas de cuidado, permitirán evitar el estrés que se alcanza cuando las demandas sobrepasan la capacidad de adaptación de la familia, y empleará herramientas y conocimientos para sustentar e incluso mejorar los cuidados de enfermería que brinda (1-3). La relación paciente-enfermera constituye el principal nexo entre las múltiples acciones que la enfermera realiza a diario, y en esa relación, el cuidado -que hace referencia a las acciones intencionales, como entregar cuidado físico y emocional o promover una sensación de seguridad, en otros- (4), muchas veces no se evidencia en el proceso de comunicación que se lleva a cabo diariamente con el paciente, e incluso ha sido descrito como un aspecto difícil y potencialmente estresante de la enfermería (5) CARE-Q. La interacción con los familiares también es una tarea difícil, pues requieren tener habilidades complejas para equilibrar las necesidades del paciente, de familiares y del personal (6). En todo momento deben estar bien informadas sobre el individuo, el paciente y su familia, para apoyarlos adecuadamente (7), y conver- tir en globales las acciones de enfermería que realiza. Desde el ingreso del paciente al hospital, las familias pueden tener muchas y variadas necesidades. Del mismo modo, la planificación de las intervenciones para atender las necesidades familiares es fundamental, ya que los miembros de las familias se han identificado como los responsables de tomar las decisiones durante la enfermedad del paciente (8). En este contexto, la existencia del modelo biomédico, que impone su lógica en la realización de múltiples tareas delegadas, frente al cuidado holístico que realiza la enfermera al momento de ejercer su profesión, genera dificultades para interactuar de forma más cercana con el paciente y su familia (9).

Teniendo en cuenta esta realidad, y sabiendo además que el cuidado es un fenómeno universal y que el aporte que realiza la cultura y las características propias de cada individuo repercuten directamente en la comprensión que se pueda tener acerca de él, la enfermera debe incorporar la diversidad del paciente y familia para entregar un cuidado acorde a cada uno de ellos (10-12). Las conductas que lleve a cabo diariamente la enfermera conformarán la percepción que tanto familiares como pacientes tengan acerca del cuidado. La visibilidad de estas conductas evitarán que su trabajo se exprese solamente a través de un modelo que impone una sobrecarga de trabajo del tipo biomédico con labores mecanizadas, que determinan que su trabajo se torne invisible (13). Las dificultades existentes, aun en la actualidad, en relación a la poca visualización y aceptación del cuidado en la práctica clínica habitual de enfermería, genera una sensación de deshumanización, que es percibida por los familiares en todos los tipos de unidades clínicas, porque observan que el manejo del equipamiento y la tecnología existente depara, aparentemente, mayor atención.

La necesidad de enfrentar esta situación, y de realizar conductas orientadas hacia la humanización de la atención, encuentra sustento en la Teoría Transpersonal de Jean Watson, 
en donde el "cuidado" es para la enfermería su razón moral, que no se definiría por un procedimiento o una acción, ya que el cuidar sería un proceso interconectado e intersubjetivo de emociones compartidas entre el profesional de enfermería y su paciente $(9,14,15)$.

En esta teoría se reconoce lo imperativo del cuidar y la necesidad de incorporar las experiencias humanas a través del desarrollo de supuestos, factores caritativos, relaciones de cuidado transpersonal y el momento del cuidado (14, 16-18). Las enfermeras no pueden asegurar que las conductas diarias, que se desarrollan habitualmente, sean percibidas de la misma manera, tanto por pacientes como por familiares, por ende las enfermeras no pueden asumir que los pacientes percibirán el esfuerzo del cuidado que ellas realizan (10). Esto se traduce en que la apreciación de cuáles conductas son consideradas relevantes, para enfermeras y pacientes, muestra diferencias, incluso diferencias marcadas en relación a qué comportamientos de cuidado son los que hacen que el paciente se sienta cuidado "por" (4).

Con todo, el cuidado en tanto imperativo moral, es un trato humano, un afecto, una interacción personal y una intervención terapéutica (19-21).

Las diferentes variaciones del concepto de cuidado y de las percepciones que se tienen de él, sumado a la ausencia de estudios a nivel nacional, hicieron necesario el desarrollo de esta investigación. Para esto se planteó el siguiente problema de investigación: ¿Existen diferencias respecto de cuáles son las conductas de cuidado importantes entre las enfermeras y los familiares de pacientes que tiene bajo su cuidado en un servicio hospitalario? Se tiene la plena convicción de que conociendo esta realidad, es decir, las percepciones propias y externas de todas aquellas conductas que generaran el concepto del cuidado, se podrán implementar cambios necesarios para una mejora continua de la práctica de enfermería.

Teniendo el sustento teórico de Jane Wat- son, el objetivo de estudio fue el siguiente: determinar si existen diferencias entre las enfermeras de pacientes hospitalizados y sus familiares, respecto de la percepción de cuáles conductas de cuidado son importantes.

Las variables de estudio fueron: conductas de cuidado consideradas importantes, edad, sexo, estado civil, nivel educacional y vínculo. En las enfermeras se midió la universidad de egreso, cursos de postgrado y años de experiencia laboral.

\section{MATERIAL Y MÉTODO}

Tipo de investigación: Investigación de tipo cuantitativa, transversal, aprobada por el Comité de Ética del hospital en donde se llevó a cabo. Población: Las unidades de análisis fueron las enfermeras y familiares de pacientes hospitalizados en unidades y servicios de un Hospital de Santiago de Chile. Se consideraron servicios en donde era posible encontrar personas hospitalizadas por más de $24 \mathrm{~h}$. El tipo de muestreo de pacientes y enfermeras fue por conveniencia. Se consideraron todos los pacientes que estuvieron hospitalizados entre marzo y julio del año 2014 en los siguientes 6 servicios: Unidad de Cuidado Intensivo Adulto, Unidad de Cuidado Intensivo Pediátrico, Unidad de Tratamiento Intermedio, Servicio Médico-Quirúrgico, Servicio de Pediatría y Sección de Hospitalizados del Servicio de Urgencia. En relación a las enfermeras, se consideraron todas las de los servicios descritos, por lo que la muestra estuvo constituida finalmente por 54 enfermeras y 96 familiares de pacientes. Criterios de inclusión: familiares co-sanguíneos directos, cónyuge o conviviente de pacientes con más de 18 años de edad y con más de 6 meses de hospitalizado; enfermeras con más de 6 meses de ejercicio profesional. Criterios de exclusión: familiares de pacientes fallecidos durante el momento de aplicación de la encuesta, como también aquellas enfermeras que desempeñaban roles 
de coordinación y/o supervisión clínica.

Luego de obtener el consentimiento informado, la recolección de información de familiares de pacientes se realizó entre marzo y julio de 2014, por una investigadora en el horario de visitas. Las entrevistas a las enfermeras se realizaron durante los días en que se acudió a entrevistar a los familiares de los pacientes seleccionados.

Las variables sociodemográficas, educacionales y laborales se midieron a través de una encuesta construida para tales efectos. La variable conductas de cuidado consideradas importantes se midió con el Caring Assessment Instrument (Care - Q) basado en la teoría de Jane Watson y elaborado por Larson (22). Este instrumento busca identificar las conductas de cuidado que las enfermeras perciben como importantes para que los pacientes se sientan cuidados. Consta de 50 preguntas tipo Likert, divididas en subescalas que indagan respecto de 6 comportamientos de la enfermera, los cuales son: a) "Es accesible", es decir, la fácil disponibilidad de tener enfermeras para el paciente y la familia, medida con 6 preguntas; b) "Explica y facilita", definida como la enseñanza, aclarar y presentar conductas de defensa hacia el paciente, compuesta de 6 preguntas; c) "Conforta", definida como la entrega de soporte físico y emocional a los pacientes y su familia, evaluada por 9 preguntas; d) "Se antici- pa", considerada como la anticipación de las enfermeras a los cambios de situación de los pacientes, tomando acciones de anticipación, compuesta de 5 preguntas; e) "Mantiene relación de confianza", es decir, la transmisión de un sentido de compromiso y comprensión para el paciente y evaluada a través de 16 preguntas; f) "Monitorea y hace seguimiento", es decir, la demostración de competencia profesional y garantía de que las acciones de enfermería delegadas a otros se finalizaron, medida con 8 preguntas. Las preguntas de las diferentes subescalas se puntúan de 1 a 5 por su importancia, donde 1 es nada importante, y 5 es muy importante.

La información recogida se ingresó a una base de datos en Epi Info 7 y se analizó con estadística descriptiva y medidas de resumen. La comparación de medias se realizó a través de la prueba $t$-Student, con una confianza del $95 \%$.

\section{RESULTADOS}

Comportamiento demográfico de la muestra: Las características demográficas de ambos grupos se resumen en la Tabla 1. En el grupo de profesionales predominan las mujeres (92,5\%) jóvenes (Media edad 32,9 DS $8,8)$ y solteras $(64,8 \%)$.

Tabla 1. Características demográficas de enfermeras y familiares.

\begin{tabular}{|c|c|c|c|c|c|}
\hline & & \multicolumn{2}{|c|}{ Enfermeras } & \multicolumn{2}{|c|}{ Familiares } \\
\hline & & $\mathrm{N}$ & $\%$ & $\mathrm{~N}$ & $\%$ \\
\hline \multirow[t]{2}{*}{ Sexo } & Masculino & 4 & 7,4 & 23 & 23,9 \\
\hline & Femenino & 50 & 92,5 & 73 & 76 \\
\hline \multirow[t]{5}{*}{ Estado Civil } & Soltero & 35 & 64,8 & 14 & 14,5 \\
\hline & Casado & 16 & 29,6 & 77 & 80,2 \\
\hline & Viudo & 0 & 0 & 1 & 1,04 \\
\hline & Divorciado & 2 & 3,7 & 3 & 3,1 \\
\hline & Anulado & 1 & 1,8 & 1 & 1,04 \\
\hline \multirow[t]{2}{*}{ Edad } & Media (DS) & \multicolumn{2}{|c|}{$32,9(8,8)$} & \multicolumn{2}{|c|}{$45(13,7)$} \\
\hline & Min/Max & \multicolumn{2}{|c|}{$24-65$} & \multicolumn{2}{|c|}{$20-75$} \\
\hline
\end{tabular}


El 58,4\% de las enfermeras estudió en universidades privadas, el resto en universidades públicas. Un 33\% de las enfermeras refiere realización de cursos de postítulo; de éstas, la mayor proporción corresponde a enfermeras de Unidades de Paciente Crítico Adulto $(9 ; 50 \%)$ y pediatría $(4 ; 22,2 \%)$. Los tipos de postítulo mayormente realizados corresponden a diplomados y especialidades, los cuales se relacionan estrechamente con sus áreas de trabajo.

En el grupo de familiares predominan las mujeres casadas de edad media, el vínculo más frecuente es de padres con un $45,8 \%$, de ellos, la presencia de la madre alcanza un $70,4 \%$, en segundo lugar se encuentra el cónyuge con un $22,9 \%$, seguido del vínculo hijos con un $10,4 \%$, abuelos $(6,2 \%)$, hermanos $(6,2 \%)$ y otros familiares $(8,3 \%)$. El nivel educacional muestra predominio de educación media con $43,7 \%$, seguido de educación técnica profesional con $23,96 \%$ y educación superior con un $20,83 \%$.

Conductas de cuidado por grupo: La percepción del cuidado muestra que en ambos grupos estudiados "Monitorea y hace seguimiento" obtiene el primer lugar de selección, con valores de media de 4,77 (DS 0,45) en el grupo de enfermeras y de 4,9 (DS 0,37) en familiares. La priorización varía según grupo estudiado, siendo las siguientes en elección para las enfermeras: "Conforta" (media 4,5, DS 0,67) y "Accesible" (media 4,82, DS 0,46). En el caso de los familiares las priorizaciones son: "Se anticipa" (media 4,82, DS 0,46) y "Accesible" (media 4,44, DS 0,87). Existe diferencia estadísticamente significativa en tres de las subescalas analizadas $(\mathrm{p}<0.001)$, estas corresponden a las categorías "Explica y facilita", "Se anticipa" y "Mantiene relación de confianza", las que se ubican en diferente lugar de importancia en los grupos (Tabla 2).

Tabla 2. Priorización de categorías de cuidado de enfermeras y familiares.

\begin{tabular}{lccccccccc}
\hline & \multicolumn{3}{c}{ Enfermeras } & \multicolumn{7}{c}{ Familiares } \\
\hline Subescala & Media & DS & Ranking & Media & DS & Ranking & $\mathrm{t}$ & $\mathrm{p}$ \\
\hline Accesible & 4,44 & 0,87 & 3 & 4,74 & 0,67 & 3 & $-0,21$ & 0,820 \\
Explica y facilita & 4,30 & 0,81 & 6 & 4,72 & 0,61 & 4 & $-3,31$ & 0,001 \\
Conforta & 4,50 & 0,67 & 2 & 4,68 & 0,63 & 5 & $-1,61$ & 0,100 \\
Se anticipa & 4,42 & 0,69 & 4 & 4,82 & 0,46 & 2 & $-3,81$ & 0,000 \\
Mantiene relación de confianza & 4,32 & 0,78 & 5 & 4,65 & 0,65 & 6 & $-2,61$ & 0,000 \\
Monitorea y hace seguimiento & 4,77 & 0,45 & 1 & 4,9 & 0,37 & 1 & $-1,8$ & 0,070 \\
\hline
\end{tabular}

$\mathrm{Al}$ analizar las 10 conductas prioritarias, según las más altas puntuaciones de medias por ambos grupos, se registraron diferencias (Tabla 3). Para las enfermeras está en primer lugar "Administra al paciente los tratamientos y medicinas oportunamente" (categoría "Accesible", media 5, DS 0). En el grupo de familiares la conducta más importante es "Sabe aplicar inyecciones, etc. y manipular equipos de uso parenteral (sueros), las máquinas de aspiración y otros equipos que hayan en el servicio" (categoría "Monitorea y hace seguimiento", media 5, DS 0). Respecto de las priorizadas, los grupos presentan diferencia estadísticamente significativa en la comparación de medias $(\mathrm{p}<0,05)$ de las siguientes conductas: "Sabe cuándo llamar al médico", "Examina al paciente con frecuencia", "Comenta con él/la paciente, en lenguaje comprensible, lo que es importante que conozca 


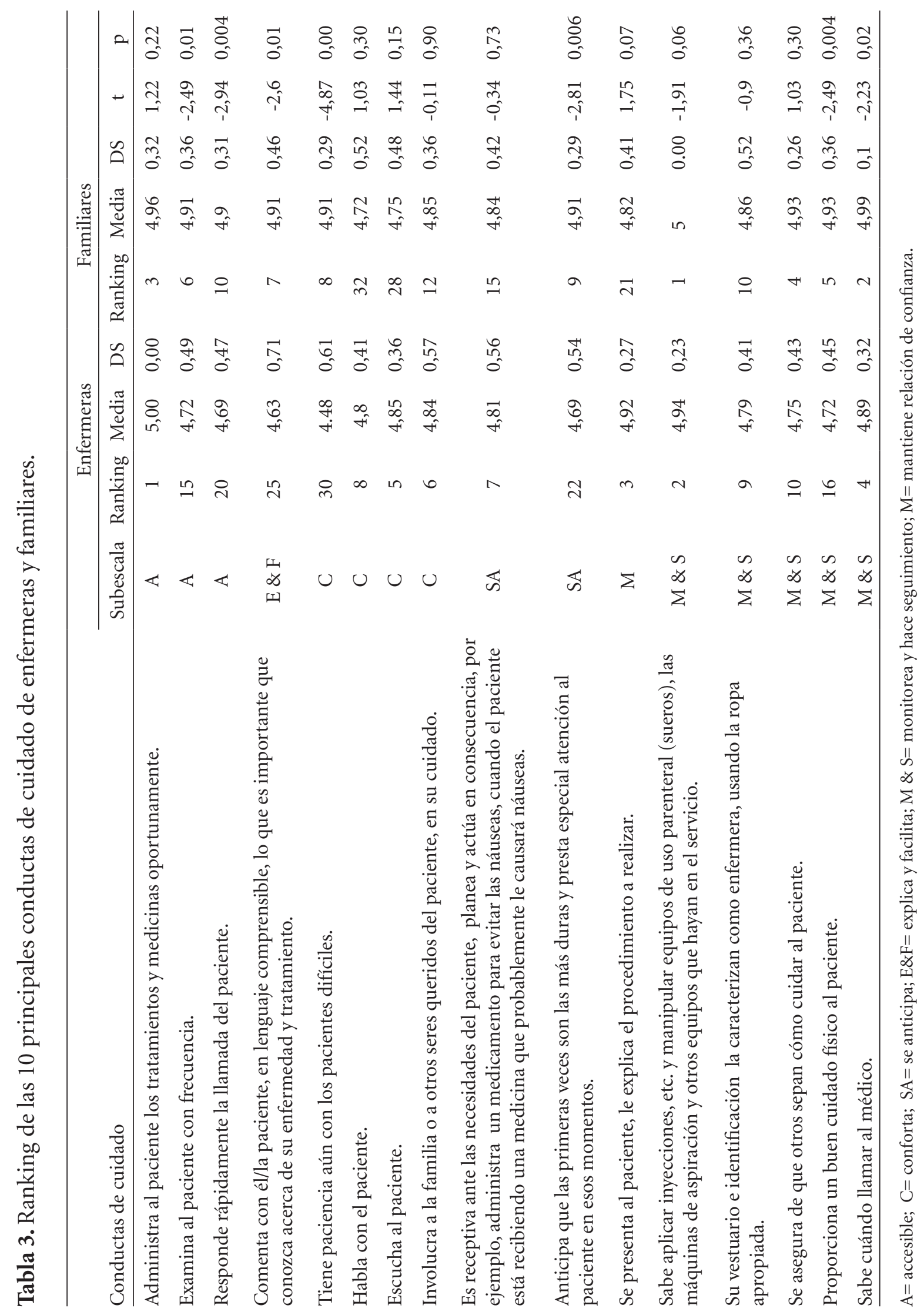


acerca de su enfermedad y tratamiento", "Anticipa que las primeras veces son las más duras y presta especial atención al paciente en esos momentos", "Responde rápidamente la llamada del paciente", "Tiene paciencia aún con los pacientes difíciles", "Proporciona un buen cuidado físico al paciente".

\section{DISCUSIÓN Y CONCLUSIÓN}

Aunque la selección de la muestra por conveniencia puede ser considerada una limitación en este estudio, creemos que este trabajo aporta una interesante visión de la percepción del cuidado de las enfermeras y familiares de pacientes, asunto no estudiado en nuestro país. La muestra de enfermeras estudiadas difiere con estudios internacionales, en donde se enfocan en grupos de enfermeras específicas de servicios puntuales. En este estudio la muestra de enfermeras abarca todas las especialidades disponibles en el hospital, lo cual entrega heterogeneidad de las respuestas y la obtención de una visión plural de las enfermeras de este hospital.

Es relevante que la ubicación de la categoría "monitorea y hace seguimiento", en este estudio, fue ubicada en primer lugar por ambos grupos. La selección de esta categoría en el grupo de enfermeras concuerda con otros estudios $(4,10)$, pero difiere de la ubicación en estudios como el de Larsson el al. (5), Larson (23), Mayer (24), Essen y Sjodén (25) y Bautista (26), donde esta categoría fue ubicada en los últimos lugares. Esta discrepancia encuentra fundamentos en la necesidad que tienen las enfermeras de entregar un cuidado caracterizado por comportamientos que evidencien su capacidad científica y técnica, con habilidades y actitudes que demuestren conocimiento, todas competencias que permiten impactar en el estado de salud del paciente y entregar cuidados con mayor seguridad. La inclusión de las unidades de mayor complejidad en este estudio, como las de paciente crítico y urgencias, que en conjunto aportaron con más del $50 \%$ de la muestra, explicarían esta tendencia a elegir conductas relacionadas con la técnica por sobre aquellas que se componen por las habilidades psicosociales. En estos servicios, la enfermera realiza, en la mayor parte de su tiempo, complejas actividades de tipo técnico, utiliza tecnología compleja dada la necesidad de monitorizar estrechamente a los pacientes, por el seguimiento propio que requiere este tipo de usuario. Este argumento también está presente en el estudio de Greenhalgh (4), en donde la presencia de enfermeras de unidades coronarias y el trabajo que allí se realiza, explicaría la elección en primer lugar de esta categoría.

Por su parte, en este estudio la categoría "conforta", ubicada en segunda posición, concuerda con el estudio de Mayer (24) en cuanto a su elección. En estudios previos (5, $23,25)$, esta categoría queda ubicada como primera opción y la variación de la posición de esta categoría es explicada en otros estudios por la presencia de un mayor número de hombres en las muestras, que no sería el caso de este estudio.

La categoría "accesible", que hace referencia a prestar cuidados orientados a dar ayuda de forma inmediata, ocupa el tercer lugar en este estudio, lo que es coincidente con otros estudios $(5,23,25)$ en donde la categoría ocupa la misma ubicación. En aquellos estudios no coincidentes $(4,10)$, esta categoría queda posicionada en los últimos lugares.

En relación a la categoría "se anticipa", los estudios realizados ubican esta conducta en lugares más altos de ponderación $(4,5,10$, 23-26). Esta diferencia es difícil de explicar, incluso considerando que la muestra se encuentra compuesta en su mayoría por unidades de mayor complejidad, en donde se esperaría que la enfermera, realizando las conductas de cuidado que contiene esta categoría, debería evitar la ocurrencia de situaciones de mayor complejidad.

En la selección de las últimas categorías 
se encuentra "mantiene una relación de confianza" y "explica y facilita", las cuales coinciden con varios estudios ya mencionados anteriormente. Cabe destacar que la elección en último lugar de la categoría "explica y facilita”, en donde la enfermera debe entregar y canalizar la información suficiente a través de la educación acerca de la condición de salud del paciente, podría explicarse por la importancia entregada a las conductas del tipo técnicas por sobre las sociales, en donde, como se ha mencionado anteriormente, la enfermera tiende a abocarse a labores derivadas del modelo biomédico.

En relación a los familiares, las mediciones realizadas a las categorías coinciden en dos de las seis categorías con el grupo enfermeras, lo que demostraría que la percepción del cuidado variaría en un grupo que la mayor parte de las veces permanece como observador. Al revisar la literatura, se observa coincidencia en la prioridad de las categorías "monitorea y hace seguimiento" y "se anticipa”. La ubicación en los últimos lugares de las categorías "conforta” y "mantiene relación de confianza" podría explicarse por la posible preferencia de los familiares hacia una enfermera orientada a las actividades técnicas más que al desarrollo de habilidades sociales que privilegian el contacto y el desarrollo de relaciones de confianza con el paciente, percibiendo así un cuidado seguro, basado también en el conocimiento que demuestra la enfermera y las técnicas que realiza. En cuanto a las conductas de cuidado estudiadas en el grupo de enfermeras, destaca que a pesar de ser considerada como más importante la categoría "monitorea y hace seguimiento", la conducta "administra los tratamientos y medicinas oportunamente" perteneciente a la categoría "accesible" se ubica en primer lugar, alcanzando la mayor media de las 50 conductas estudiadas. Para otros estudios $(22,24,25)$ esta conducta no es prioritaria, con excepción del estudio de Zamanzadeh et al. (10), en donde esta conducta también se considera como la primera elección.
La segunda conducta mencionada "sabe aplicar inyecciones, etc. y manipular equipos de uso parenteral (sueros), las máquinas de aspiración y otros equipos que hayan en el servicio" perteneciente a la categoría "monitorea y hace seguimiento", coincide con el estudio de Zamanzadeh et al. (10), en donde queda ubicado en la misma posición, sin embargo en el estudio de Larsson et al. (5) esta conducta también se menciona dentro de las cuatro primeras posiciones.

La conducta "sabe cuándo llamar al médico" posicionada en cuarto lugar, encuentra similitud en ubicación en el estudio de Larsson et al. (5). Destaca que dentro de la selección de las enfermeras, la conducta "escucha al paciente" se ubica en quinto lugar, a diferencia de todos los estudios mencionados, en donde se encuentra en el primer lugar de selección. Esta diferencia de percepción del cuidado depara mayor atención ya que habla de no considerar sentimientos y preferencias del paciente. La inclinación hacia la selección de categorías técnicas y conductas propias de ellas deberá ser considerada.

Para el grupo de familiares no existe coincidencia en la ubicación de las conductas estudiadas, variando en posición con respecto a las enfermeras. Se aprecian conductas que para los familiares son más importantes, como es el caso de "se asegura de que otros sepan cómo cuidar al paciente", que se encuentra en cuarta posición, y para las enfermeras ocupa el número 10.

Los resultados obtenidos en este estudio muestran que las categorías y conductas de cuidado son diferentes para las enfermeras y los familiares de los pacientes, evidenciado en las diversas posiciones de elección observadas en este estudio.

Las necesidades de cuidado se van orientando según el concepto de la percepción del cuidado que exista y acerca de quienes lo representan. Sus conductas muestran que la presencia de factores externos, como son la cultura y las costumbres, influiría en la percepción que se tiene acerca de él. Es impor- 
tante considerar, además, la formación de las enfermeras en las universidades, en donde el concepto del cuidado propio de la profesión se ve desplazado hacia el cumplimiento de actividades técnicas derivadas del modelo biomédico, disminuyendo el contacto con el paciente y sus familiares, parte importante del cuidado y base de la profesión.

Existe una clara necesidad de continuar con esta vertiente de estudio, que permitirá generar una imagen acerca de las necesidades de cuidado, no sólo del paciente, sino que también de los familiares y del equipo de salud.

\section{REFERENCIAS}

1. Vázquez M, Eseverri M. The caring of family members in the intensive care units from the Jean Watson perspective. Enferm Intensiva. 2010; 21(4): 161-4.

2. Pinedo M, Rebolledo D, González J. Cuidados de enfermería en el "sufrimiento espiritual” aplicando el modelo de Jane Watson. Salud, Arte y Cuidado [Internet]. 2009 Jul-Dic [citado 26 feb 2014]; 2(2): 8-13. Disponible en: http://bibvirtual.ucla.edu.ve/db/psm_ucla/edocs/sac/ sac0202/sac020203.pdf.

3. Pryzby BJ. Effects of nurse caring behaviours on family stress responses in critical care. Intensive Crit Care Nurs. 2005; 21(1): 16-23.

4. Greenhalgh J, Vanhanen L, Kyngäs H. Nurse caring behaviours. J Adv Nurs. 1998; 27(5): 927-32.

5. Larsson G, Widmark V, Lampic C, von Essen L, Sjödén PO. Cancer patient and staff ratings of the importance of caring behaviors and their relations to patient anxiety and depression. J Adv Nurs. 1998; 27(4): 855-64.

6. Agård AS, Harder I. Relatives' experiences in intensive care-finding a place in a world of uncertainty. Intensive Crit Care
Nurs. 2007; 23(3): 170-7.

7. Agård AS, Maindal HT. Interacting with relatives in intensive care unit. Nurses' perceptions of a challenging task. Nurs Crit Care. 2009; 14(5): 264-72.

8. Stayt LC. Nurses' experiences of caring for families with relatives in intensive care units. J Adv Nurs. 2007; 57(6): 62330.

9. Poblete M, Valenzuela S, Merino J. Validación de dos escalas utilizadas en la medición del cuidado humano transpersonal basadas en la Teoría de Jean Watson. Aquichan. 2012; 12(1): 8-21.

10. Zamanzadeh V, Azimzadeh R, Rahmani A, Valizadeh L. Oncology patients' and professional nurses' perceptions of important nurse caring behaviors. BMC Nurs [Internet]. 2010 Jun [citado 31 dic 2014]; 9: 10. Disponible en: http:// www.pubmedcentral.nih.gov/articlerender.fcgi?artid=2902470\&tool=pmcentrez\&rendertype $=$ abstract.

11. Holroyd E, Cheung Y, Cheung S, Luk F, Wong W. A Chinese cultural perspective of nursing care behaviours in an acute setting. J Adv Nurs. 1998; 28(6): 1289-94.

12. Rexroth R, Davidhizar R. Caring: utilizing the Watson theory to transcend culture. Health Care Manag (Frederick). 2003; 22(4): 295-304.

13. Poblete M,Valenzuela S.Cuidadohumanizado: un desafío para las enfermeras en los servicios hospitalarios. Acta Paul Enferm [Internet]. 2007 Dec [citado 22 feb 2014]; 20(4): 499-503. Disponible en: http:// www.scielo.br/scielo.php?script $=$ sci_arttext\&pid=S0103-21002007000400019\&1$\mathrm{ng}=\mathrm{es} \& \mathrm{nrm}=\mathrm{iso} \& \mathrm{t} \operatorname{lng}=\mathrm{es}$.

14. Sitzman KL. Teaching-Learning Professional Caring Based on Jean Watson's Theory of Human Caring. Int J Hum Caring. 2007; 11(4): 8-16.

15. Favero L, Paqliuca LM, Lacerda MR. Cuidado transpessoal em enfermagem: uma análise pautada em modelo conceitual. Rev Esc Enferm USP. 2013; 47(2): 500-5. 
16. Urra E, Jana A, García M. Algunos aspectos esenciales del pensamiento de Jean Watson y su teoría de cuidados transpersonales. Cienc. enferm. 2011; XVII(3): 11-22.

17. Cossette S, Cara C, Ricard N, Pepin J. Assessing nurse-patient interactions from a caring perspective: report of the development and preliminary psychometric testing of the Caring Nurse-Patient Interactions Scale. Int J Nurs Stud. 2005; 42(6): 673-86.

18. Lukose A. Developing a practice model for Watson's theory of caring. Nurs Sci Q. 2011; 24(1): 27-30.

19. Beck CT. Quantitative measurement of caring. J Adv Nurs.1999; 30(1): 24-32.

20. Bailey DN, Watson J, Swanson K, Roach SS, Leininger M, Boykin A, et al. Caring Defined: A Comparison and Analysis. Int
J Hum Caring. 2009; 13(1): 16-32.

21. Caro S. Enfermería: Integración del cuidado y el amor. Una perspectiva humana. Salud Uninorte. 2009; 25(1): 172-178.

22. Larson P. Important nurse caring behaviors perceived by patients with cancer. Oncol Nurs Forum. 1984; 11(6): 46-50.

23. Larson P. Cancer nurses' perceptions of caring. Cancer Nurs. 1986; 9(2): 86-91.

24. Mayer D. Oncology nurses versus cancer patients' perceptions of nurse caring behaviours: a replication study. Oncol Nurs Forum. 1987; 14(3): 48-52.

25. Essen L, Sjodén P-O. Patient and staff perceptions of caring: review and replication. J Adv Nurs. 1991; 16(11): 1363-74.

26. Bautista L. Percepción de la calidad del cuidado de enfermería en la ESE Francisco de Paula Santander. Aquichan. 2008; 8(1): 74-84. 